\title{
Automatic generation of tower defense levels using PCG
}

\begin{tabular}{|c|c|c|}
\hline & Full Paper & \\
\hline $\begin{array}{c}\text { Simon Liu } \\
\text { LevelupAI } \\
\text { liucheng@levelup.ai }\end{array}$ & $\begin{array}{c}\text { Li Chaoran } \\
\text { LevelupAI } \\
\text { lichaoran@levelup.ai }\end{array}$ & $\begin{array}{c}\text { Li Yue } \\
\text { LevelupAI } \\
\text { liyue@levelup.ai }\end{array}$ \\
\hline $\begin{array}{c}\text { Ma Heng } \\
\text { LevelupAI } \\
\text { maheng@levelup.ai }\end{array}$ & $\begin{array}{c}\text { Hou Xiao } \\
\text { LevelupAI } \\
\text { houxiao@levelup.ai }\end{array}$ & $\begin{array}{c}\text { Shen Yiming } \\
\text { LevelupAI } \\
\text { shenyiming@levelup.ai }\end{array}$ \\
\hline $\begin{array}{c}\text { Wang Licong } \\
\text { LevelupAI } \\
\text { wanglicong@levelup.ai }\end{array}$ & $\begin{array}{c}\text { Chen Ze } \\
\text { LevelupAI } \\
\text { chenze@levelup.ai }\end{array}$ & $\begin{array}{c}\text { Guo Xianghao } \\
\text { LevelupAI } \\
\text { guoxianghao@levelup.ai }\end{array}$ \\
\hline $\begin{array}{c}\text { Lu Hengtong } \\
\text { Beijing University of Posts and } \\
\text { Telecommunications } \\
\text { luhengtong@levelup.ai }\end{array}$ & $\begin{array}{c}\mathrm{Du} \mathrm{Yu} \\
\text { Beijing University of Posts and } \\
\text { Telecommunications } \\
\text { duyu@levelup.ai }\end{array}$ & $\begin{array}{c}\text { Tang Qinting } \\
\text { Beijing University of Posts and } \\
\text { Telecommunications } \\
\text { tangqinting@levelup.ai }\end{array}$ \\
\hline
\end{tabular}

\begin{abstract}
Tower defense is a popular subgenre of real-time strategy game requiring detailed level design and difficulty balancing to create an enjoyable player experience. Because level production and testing are both time-consuming and labor-intensive, we propose and implement a framework to automate the process. We first analyze the three main components, or "building blocks", of the popular tower defense game Kingdom Rush: Frontiers (KRF), i.e. road maps, tower locations and monster sequences. We then automatically create new building blocks in the style of the original game, utilizing techniques from Procedural Content Generation (PCG), and assemble them to create new levels. We also add a fourth block: automated testing via a Monte Carlo search algorithm, to ensure the generated content is playable. We focus on KRF because it is a popular video game in the tower defense genre, and highlights some of the challenges of designing appropriate PCG and playtesting algorithms for a commercial video game.
\end{abstract}

\section{CCS CONCEPTS}

- Computing methodologies $\rightarrow$ Randomized search; Heuristic function construction; Game tree search; • Applied computing $\rightarrow$ Computer games;

\section{KEYWORDS}

computer games, PCG, tower defense, reinforcement learning

Permission to make digital or hard copies of part or all of this work for personal or classroom use is granted without fee provided that copies are not made or distributed for profit or commercial advantage and that copies bear this notice and the full citation on the first page. Copyrights for third-party components of this work must be honored. For all other uses, contact the owner/author(s).

FDG '19, August 26-30, 2019, San Luis Obispo, CA, USA

(c) 2019 Copyright held by the owner/author(s).

ACM ISBN 978-1-4503-7217-6/19/08.

https://doi.org/10.1145/3337722.3337723
ACM Reference Format:

Simon Liu, Li Chaoran, Li Yue, Ma Heng, Hou Xiao, Shen Yiming, Wang Licong, Chen Ze, Guo Xianghao, Lu Hengtong, Du Yu, and Tang Qinting. 2019. Automatic generation of tower defense levels using PCG: Full Paper. In The Fourteenth International Conference on the Foundations of Digital Games (FDG '19), August 26-30, 2019, San Luis Obispo, CA, USA. ACM, New York, NY, USA, 9 pages. https://doi.org/10.1145/3337722.3337723

\section{INTRODUCTION}

In this paper, we apply techniques from academic AI research to increase the efficiency and reliability of routine processes in commercial game development. Single-player casual games, which we focus on in our research, can require hundreds or thousands of elaborate level designs that increase in difficulty to match improvements in the player's skill. Testing each level to ensure it is playable is also time-consuming and laborious, often requiring human players. For example, the popular Candy Crush Saga has over 4,000 levels in its web edition, with around 4-5 new levels added every day[5], and has begun using AI that imitates human players to automate playtesting.[12].

While PCG can be applied to a wide variety of game genres and game assets (e.g. music, artwork, level design), we focus in this paper on level design and testing for a commercially-successful tower defense game, Kingdom Rush: Frontiers (KRF). We break down a KRF level into its constituent building blocks, as detailed in Section 1.2. For each building block, we generate new content and assemble the blocks together to form a complete new level. We choose to focus on KRF because its level of complexity is reflective of a modern casual video game, but does not require excessive computing power for us to run simulations of the game and create content for it. Although automated content creation is our focus in this paper, we also use a reinforcement learning agent to automatically learn winning strategies for each level (to confirm that the level can be won and the declared goal of the game can be achieved).

We structure this paper as follows: 


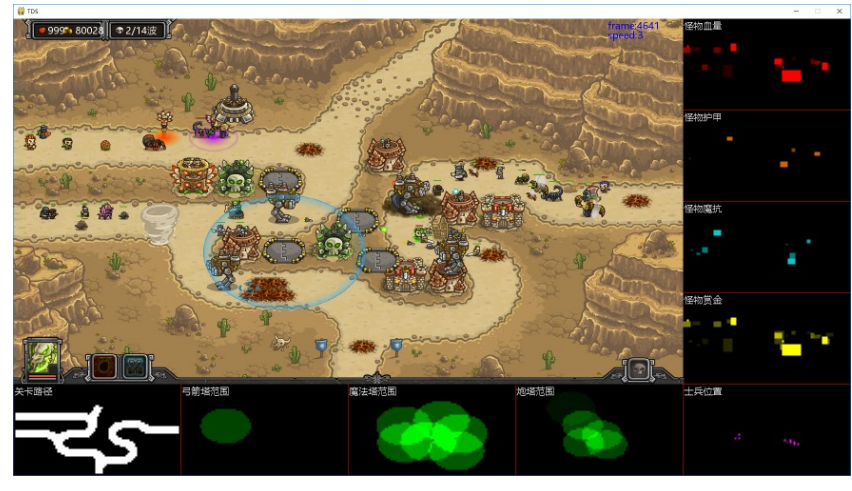

Figure 1: A screenshot of the tower defense simulator. The dashboard panels along the bottom and right-hand sides show locations of roads and other representations of the game state.

- In the remainder of this section, we discuss a tower defense game and its basic building blocks.

- Section 2 references some existing literature that puts our research into context

- In Section 3, we describe algorithms used to automatically generate levels and playtest our content.

- In Section 4, we present and discuss our results, including three algorithmically-generated levels and associated difficulty scores.

- Section 5 offers concluding remarks.

\subsection{Tower Defense Game Rules}

We use a tower defense (TD) game developed in-house as our test subject (see Figure 1), although our general approach can be extended to other single-player video games as well. Tower defense is a popular genre for casual gamers and is a proven testbed for research into AI and games[2]. For example, [1] investigates a genetic algorithm to evolve increasingly difficult monster waves for a TD game, [22] studies how to adjust a TD game's parameters dynamically based on a player's performance in previous levels, and [11] evolves automated controllers for playing a TD game.

Our testbed is modeled on the popular video game Kingdom Rush: Frontiers (KRF), released in 2013 by Ironhide Studios. KRF is a single-player game in which the goal is to defeat an army of invading monsters by strategically placing defensive towers on the game's map and micromanaging certain aspects of battle, such as the placement of reinforcement soldiers or the Rain of Fire, a powerful aerial weapon. The player initially has a limited amount of gold with which to buy and upgrade towers, but can earn more gold by killing monsters. Monsters that successfully pass through the exit points on the map will deduct lives from the player's lives count (which is initially 20). The level ends when all enemies are defeated or the player's lives count is reduced to 0 . In the original KRF, the level maps and monster waves are static, fixed in advance by the level designers. There are a small number of "Endless Challenge" levels, in which the monster waves are procedurally generated, but the amount of playable content for games in the KR video game

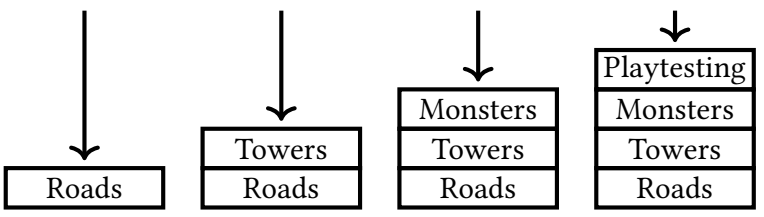

Figure 2: A graphical depiction of the four basic building blocks used to construct and test a tower defense level.

series, while high quality, is limited, which was one motivating factor for this research.

\subsection{Building Blocks of a Tower Defense Level}

We define four basic building blocks that must be realized and assembled to create a Tower Defense level in the style of KRF (see Figure 2 ), namely road maps, tower locations, monster sequences, and automated playability testing. We first disassemble levels from the original KRF game and analyze them, then apply automated methods to generate content in a similar style and perform playtesting. For simplicity, we do not consider elements such as hero characters and non-campaign play modes, which are not essential to playing the game.

1.2.1 Road maps. The foundation for any level is a set of roads crossing the map. Monsters enter from one end of a road and proceed toward the other (flying monsters also typically follow these paths). Road maps are pieced together from smoothly curved and straight line segments.

1.2.2 Tower locations. These locations, also called strategic points in the game, are fixed locations along the roadside at which the player may construct defensive towers. Unlike some TD games that allow towers to be placed freely in any location that is not on a road, $\mathrm{KRF}$ is more restrictive. We also use this style in our game.

1.2.3 Monster sequences. The most crucial element of a level is appropriate monster sequences for the player to face in battle. In $\mathrm{KRF}$, the monster sequences and the times at which monsters appear are fixed in advance by the game's designers, and are not subject to dynamic difficulty adjustment. We also follow this approach in our PCG methods.

1.2.4 Automated playability testing. We add a fourth building block to the successful generation of a TD level: that of using a reinforcement learning agent to automatically playtest the level and ensure that it is properly designed.

\section{BACKGROUND \& RELATED RESEARCH}

The algorithmic generation of content for games is referred to as Procedural Content Generation (PCG). PCG is a broad field that can automate creation of many types of content, including graphics, music and sounds, story scripts, game rules, and so on. A summary of recent PCG research may be found in [18]. A comprehensive survey of AI and games research[25] also discusses PCG at length. In this paper, we focus on a specific problem in PCG research: that of generating new levels for a tower defense game in the style of existing content, and producing a quantitative measure 
of each level's difficulty in a completely automated fashion. We first review some of the existing literature, and introduce three relevant approaches to PCG (generate and test, search-based PCG, and PCGML), as well as automated testing.

PCG has been successfully used in existing tower defense games. Examples include the independent games Dead Ground[20], which procedurally generates every level; Tronix Defender[14], which randomizes elements of each level to increase replayability; and gGuardian[10], which also uses PCG technqiues to create new level maps. In these examples, level content is generated without human intervention, which is also the approach we pursue. An alternative approach is mixed-initiative design, where a computer program generates each level based on interaction with a human designer, as in the Tanagra design tool for 2D platform games[19], the Sentient Sketchbook[16] for map creation, and the "Explainable PCGML" system[13] that outputs Super Mario Bros. levels based on examples of features specified by a human.

\subsection{Generate and Test}

One often-used paradigm in PCG is generate-and-test, where a candidate solution (i.e. level content, such as a level map) is produced by applying rules to a sequence of pseudorandom numbers (e.g. place a new square-shaped room of a given size in a given location). Rule-based tests are used to ensure the generated content conforms to certain restrictions imposed by the game's logic (e.g. two rooms in a dungeon may not overlap), and the content can be partially or completely regenerated until all the relevant tests are passed. A notable early example of this approach is the interstellar trading game Elite (1984), which used pseudorandom numbers to generate the configuration of stars and other variables in the player's universe. However, Elite's pseudorandom seed, and hence the generated universe, was fixed in advance, effectively making PCG a form of lossless data compression rather than a continual source of new content. A more recent example is the galactic exploration game No Man's Sky (2015), which used pseudorandom numbers to generate solar systems and the details of individual planets (including terrain, flora and fauna), and even aspects of the game's soundtrack[9].

\subsection{Search-based PCG}

An extension of generate-and-test is search-based PCG. This is an iterative method that generates many candidate solutions, using an evaluation function to assess which ones are most suitable according to criteria defined by a human designer. Successive iterations build on the best solutions from previous iterations until the generated result is of good quality. For example, TrackGen[4] used an evolutionary algorithm to produce race tracks for two 3D car racing games, TORCS and Speed-Dreams, ensuring that each generated track had an acceptable length and number of turns. Search-based methods are suitable when rules for generating content are difficult to define explicitly, but an evaluation function for various candidate solutions can be provided to select the most suitable ones. A multi-objective optimization method, such as a genetic algorithm or simulated annealing, may then be used to find suitable candidates from a solution space. However, the search process can be time-consuming, meaning that it may not be feasible for content that needs to be generated on demand.

\subsection{PCG via Machine Learning (PCGML)}

A third and relatively new approach is PCG via Machine Learning (PCGML)[21], which uses machine learning or related statistical techniques to analyze existing game content and extract usable parameters from them. A simple example would be to count the proportion of different elements that appear in a match-three game and imitate this statistical distribution in a newly generated level. A more sophisticated example[24] used two neural networks in a Generative Adversarial Network to generate a new Super Mario Bros. level in the style of an existing one. However, creating playable and sufficiently diverse content using pure PCGML is challenging. One difficulty is that game levels must respect certain structural constraints that are difficult to specify in a machine learning framework (e.g. all roads on a map must be linked). A second difficulty is that the amount of training data for most games is usually very small. However, some recent work in this area[15] creates new content based on a single training example.

\subsection{Playability testing}

Although not strictly part of content generation itself, simulationbased testing of PCG content can also be considered an important part of automated game content production. Previous examples include the level generator Ropossum, which uses an agent to verify puzzles are solvable in the physics-based game Cut The Rope[17]; agent-based testing of the board game Ticket to Ride[7]; and agentbased testing of a simplified version of the simulation game The Sims Mobile[6].

A general approach to automated game-playing is reinforcement learning, where a computer program plays a game without human guidance by selecting actions according to some strategy, often randomly at first, but improves its performance over time by collecting data about which actions lead to more positive rewards (as defined by an increasing game score or some other evaluation function) and adjusting its strategy accordingly in future attempts. A more comprehensive overview of reinforcement learning may be found in [23]. In this paper, we make use of Monte Carlo search, which is a planning-based technique frequently used in reinforcement learning. Reinforcement learning has also begun to see use within commercial game development[8], and we pursue this approach in our research.

\section{METHODS FOR LEVEL GENERATION AND TESTING}

Level generation for our TD game has three phases: road generation, tower point placement, and monster sequence generation. For each PCG level, we use a human-designed level from the original game as a reference dataset for our content generation. We select PCG algorithms that are simple to implement and understand, have acceptable runtime performance, and empirically produce good results. While the number of lives and amount of gold the player starts with can be very influential on difficulty, adjusting these two variables is trivial and does not require PCG techniques; therefore, 
we omit them from discussion and use values from the humandesigned levels. An investigation into how these parameters affect level difficulty and playability is a potential topic for future research.

\subsection{Road Generation}

A map is made up of individual roads that cross the map from an entrance to an exit, both typically located on the map's edge. Roads may partially overlap with each other; for example, two roads may start from different entrances but share the same exit. Each road is composed of straight line or smoothly curved segments linked together. We generate an initial road by selecting a random point near the map's center and adding linked, random road segments toward the map's edges in opposite directions. If the map requires additional roads, a random point is chosen approximately halfway along the initial road and a half-road is generated from this point toward a map edge in an unused direction; the other half of the new road is copied from the initial road, ensuring that the two roads will merge. The process is repeated until all roads have been drawn. This algorithm produces maps quickly that are similar in style to those from the original game. However, we ignore infrequent cases in the original game where maps contain roads that do not meet or where entrances and/or exits are not on the map's edges.

\subsection{Tower Point Generation}

We use random search to generate tower points alongside the generated roads. We start by placing two to three points in the vicinity of every road junction, then place the remaining points randomly on the map within a certain distance of the roadside (70 to 90 pixels from the center of a road), ensuring that no two points are too close to (within 60 pixels of) each other. To validate our generated points, we also compute the road coverage distribution of the generated tower points $\left(C_{\mathrm{pcg}}\right)$. This describes the percentage of road area within firing range of no towers, 1 tower, 2 towers, and so on. The algorithm compares this with the coverage distribution for the reference level $\left(C_{\text {ref }}\right)$ using their Kullback-Leibler $(\mathrm{KL})$ divergence:

$$
D_{K L}\left(C_{\mathrm{pcg}} \| C_{\mathrm{ref}}\right)=\sum_{i} C_{\mathrm{pcg}}(i) \log \frac{C_{\mathrm{pcg}}(i)}{C_{\mathrm{ref}}(i)}
$$

The algorithm keeps generating new candidate tower point sets until the KL divergence of the two distributions is below an acceptable threshold ( 0.1 in our experiments). This random search-based method is extremely fast and produces good results in practice.

\subsection{Monster Generation}

3.3.1 Cluster extraction from original game. We first extract clusters of monsters that appear together in the original levels: a cluster is defined as one or more monsters appearing from the same entrance, separated from the previous and next monster by no more than five seconds (see Figure 3). We use these clusters, rather than individual monsters, as the basic alleles in our genetic representation to reduce the amount of computation required.

3.3.2 Genetic representation. A level is divided into several time stages called waves. Each wave consists of monsters following various roads on the map toward an exit. In each wave, we use a

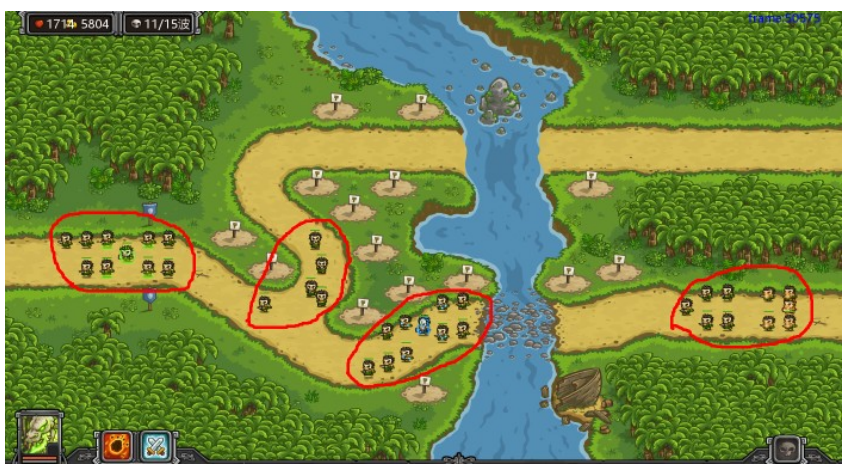

Figure 3: An example generated monster sequence. "Clusters" of monsters that appear together are highlighted.

fixed-length chromosome (see Fig. 4) to represent a monster sequence for a particular wave on a given road. Each gene is a numeric ID representing a particular monster cluster from the original game. The chromosome is fixed in length to facilitate easier processing, but a gene may also be empty, which corresponds to no monsters appearing; this means that waves can be of different lengths in the actual game level, with the fixed length acting as an upper bound.

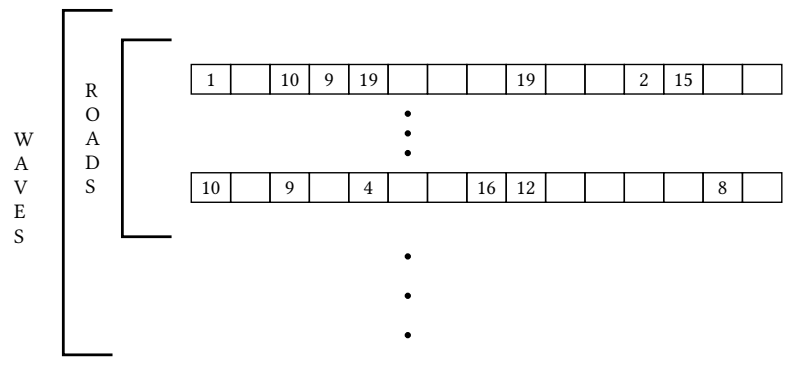

Figure 4: The genetic representation used to evolve monster sequences. Each chromosome contains clusters of monsters extracted from the original game. Because each level has multiple waves and roads, the complete candidate sequence is a list of chromosome lists.

3.3.3 Fitness function. We randomly generate waves using the monster clusters extracted from the original game. The initial population of monster sequences is then evolved using a genetic algorithm. Our goal is to generate monster sequences $\left(s_{1}, \ldots, s_{n}\right)$ that are similar in character to that of the original level $\left(s_{\text {ref }}\right)$, so our fitness function measures the similarity of candidate sequences to $s_{\text {ref }}$ along three dimensions:

(1) Monster count, where $N_{c}$ and $N_{r}$ are lists representing the number of monsters per wave in a candidate level and reference level respectively. $N_{c i}$ and $N_{r i}$ are the number of monsters in the $i$ th wave of the given level.

(2) Monster count by type, $T_{c}$ and $T_{r}$, represent the number of monsters of each type in a candidate and reference level. $T_{c i}$ and $T_{r i}$ are the counts for the $i$ th wave of a level. $T^{\prime}$ is the number of different enemy types for the entire level. 
(3) Total hit points, $H_{c}$ and $H_{r}$, represents the total number of hit points for all monsters in a candidate or reference level $H_{c i}$ and $H_{r i}$ are the total hit points of all monsters in the $i$ th wave of a level.

To measure similarity for each feature, we first define two distance metrics to quantify the similarity between two sequences, $s_{1}$ and $s_{2}$ :

$$
\begin{gathered}
d_{1}\left(s_{1}, s_{2}\right)=\sum_{i}\left|s_{1 i}-s_{2 i}\right| \\
d_{2}\left(s_{1}, s_{2}\right)=1-\frac{\sum_{i} s_{1 i} s_{2 i}}{\sqrt{\sum_{i}\left(s_{1 i}\right)^{2}} \sqrt{\sum_{i}\left(s_{2 i}\right)^{2}}}
\end{gathered}
$$

Eq. 2 comes from Manhattan Distance and Eq. 3 from cosine similarity. These are then used to define three fitness evaluation functions:

$$
\begin{gathered}
f_{N}=d_{1}\left(N_{c}, N_{r}\right)+d_{2}\left(N_{c}, N_{r}\right)+\left|\sum N_{c}-\sum N_{r}\right| \\
f_{T, T^{\prime}}=\theta_{1} d_{1}\left(T_{c}, T_{r}\right)+\theta_{2}\left|T_{c}^{\prime}-T_{r}^{\prime}\right| \\
f_{H}=\theta_{3} d_{1}\left(H_{c}, H_{r}\right)+\theta_{4} d_{2}\left(H_{c}, H_{r}\right)
\end{gathered}
$$

where $\theta_{n}$ is a weight parameter $\left(\theta_{1}=50, \theta_{2}=100, \theta_{3}=1\right.$, $\left.\theta_{4}=50\right)$. Overall fitness value is a linear combination of the three functions above:

$$
\text { fitness }=f_{N}+f_{T, T^{\prime}}+f_{H}
$$

3.3.4 Generation. The genetic algorithm proceeds through four stages repeatedly until a maximum number of iterations is reached or population fitness converges.

- Calculate fitness of all candidates in population $P$.

- Select elite: top $20 \%$ of individuals in $P$ pass through to next stage unchanged.

- Produce children: The remaining $80 \%$ of individuals in the new population are produced by mating the top $20 \%$ from the previous one.

- Mutate children. $30 \%$ of the elite candidates' children are randomly mutated.

We chose the hyperparameters listed above after a search for the most effective ones (see Figure 5).

\subsection{Agent-based playtesting}

After the level has been generated, an automated game-playing agent starts to play the game. Every 50 frames, the game simulator provides a list of available actions to the agent. If the action list has not changed in the previous 50 frames, we skip this decision automatically to reduce our search space. Typically, there will be between 10-100 different actions that can be chosen.

Available actions are provided to the agent as follows: we first tile the game map into an $88 \times 48$ grid; our algorithm can then play the game like a human by "clicking" on the screen at a particular gridcell. To reduce unnecessary computation, our game simulator filters out a large number of gridcells at each decision step where a click would not result in any change to the game's state. For the same reason, we also forbid our algorithm from selling its towers.

Our reinforcement learning agent uses a variant of Monte Carlo search (flat Monte Carlo) to assess the value of each action. Flat Monte Carlo is a simpler variant of the widely-used Monte Carlo

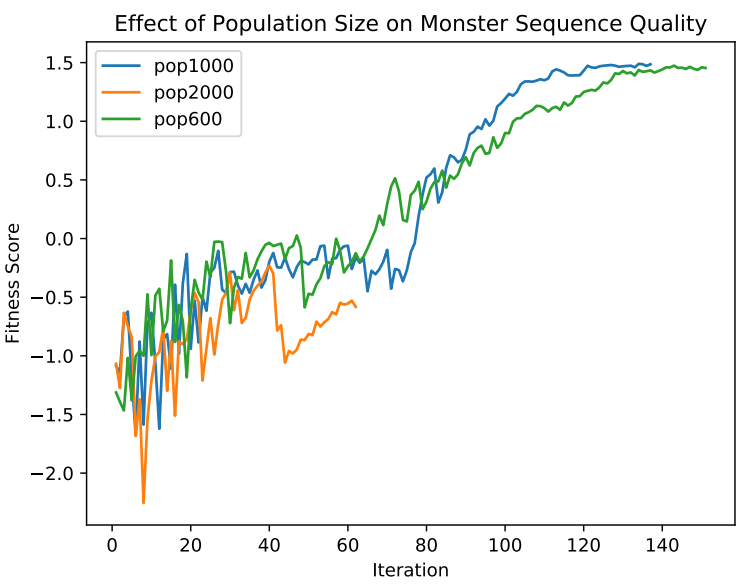

Figure 5: Effect of population size (a hyperparameter) on the genetic algorithm's convergence. A population size of 2,000 candidates in each generation results in faster convergence, but the final fitness is poor in comparison to smaller population sizes. We chose to use a population size of 1,000 after a hyperparameter search.

Tree Search (MCTS) [3], as we simply start a fixed number of Monte Carlo rollouts from each possible action that can be chosen in the current state; there is no tree of statistics to guide where the agent should focus its searches. We choose flat Monte Carlo search because it is easier to run in parallel (and therefore faster) and uses less memory than MCTS, and achieves good results in practice. A manually defined evaluation function assesses the outcome of each rollout and backpropagates the result to the current state. The evaluation function considers:

(1) The percentage of total enemies killed so far, $P_{\boldsymbol{e}}$.

(2) The percentage of player lives (hearts) remaining, $P_{h}$.

(3) A number classifying the average damage dealt by the Rain of Fire each time it has been used in this level, denoted $N_{f}$. If the average damage is over 3,000 hit points, then the number is set to 1 , otherwise it is 0 .

(4) A number classifying the proportion of time that reinforcements spend fighting enemies as opposed to standing idle, denoted $N_{r}$. If the percentage is more than $70 \%$, then the number is set to 1 , otherwise it is 0 .

The first two elements reflect to what extent the player has fulfilled the main strategic objective of the game (killing enemies), while the latter two elements are related to the player's success in tactical micromanagement. The evaluation function $G(s)$ of state $s$ is a linear composition of the elements described above.

In our experiments, $G(s)$ is defined as in Eq.5, where the coefficients are empirically chosen.

$$
G(s)=0.76 * P_{e}+0.2 * P_{h}+0.02 *\left(N_{f}+N_{r}\right)
$$

After all rollouts are complete, the agent chooses the action leading to the highest-valued state. By fixing the pseudorandom seed used in our simulations, we can create a completely deterministic 


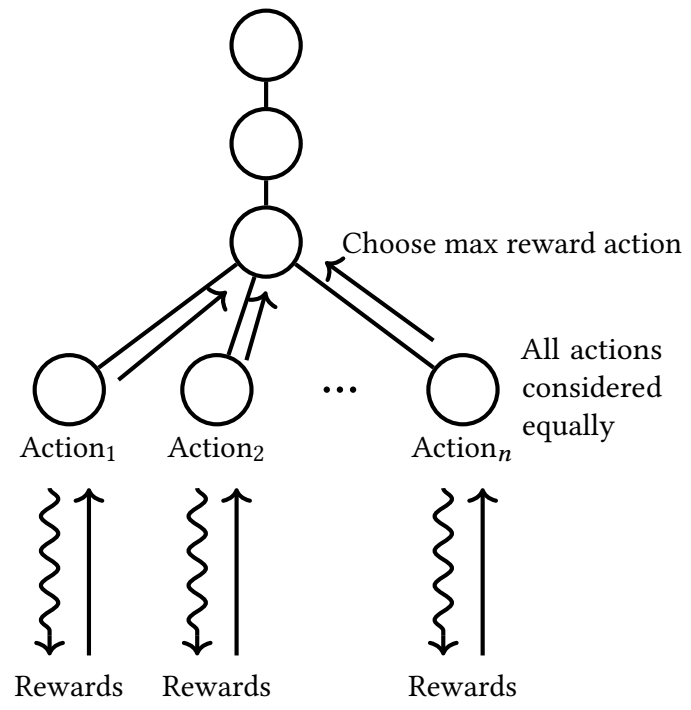

Figure 6: Graphical representation of flat Monte Carlo Search. Flat Monte Carlo differs from the popular MCTS in that it does not use statistics collected from previous searches to direct its exploration along more promising trajectories. This makes flat Monte Carlo less effective generally, but with the benefits that it is quicker and more memory-efficient.

model of the environment for lookahead search, and therefore do not need to account for uncertainty by choosing an average value.

At each state where a decision can be made, we define the current perceived difficulty of the level as the weighted sum of two factors. One factor is the variance in action values after lookhead search: if the variance is low, then it does not matter much which action the agent chooses, and the game state is considered to be easy; we describe this as strategic difficulty $\left(D_{S}\right)$. The second factor, $D_{G}$, is based on a goal-completeness metric, defined as what proportion of enemies have already been killed. In early stages of the game, when most enemies are still alive or have yet to enter the map, this proportion is low and difficulty is high, in an inversely proportional relationship. We consider early stages of the level to be more difficult than later stages because there are more decisions remaining for the player to make, and uncertainty of outcome (whether the player will win or lose) is higher. The difficulty at time $t$ is defined as:

$$
D^{t}=D_{S}^{t}+D_{G}^{t}
$$

Our difficulty rating varies over time, but we can calculate the total difficulty $D$ for an entire level as the sum of all difficulty scores. Levels where more decisions need to be made therefore tend to be assessed as more difficult.

$$
D_{\text {level }}=\sum_{t=0}^{T}\left(D_{S}^{t}+D_{G}^{t}\right)
$$

A graphical description of flat Monte Carlo search is given in Figure 6.
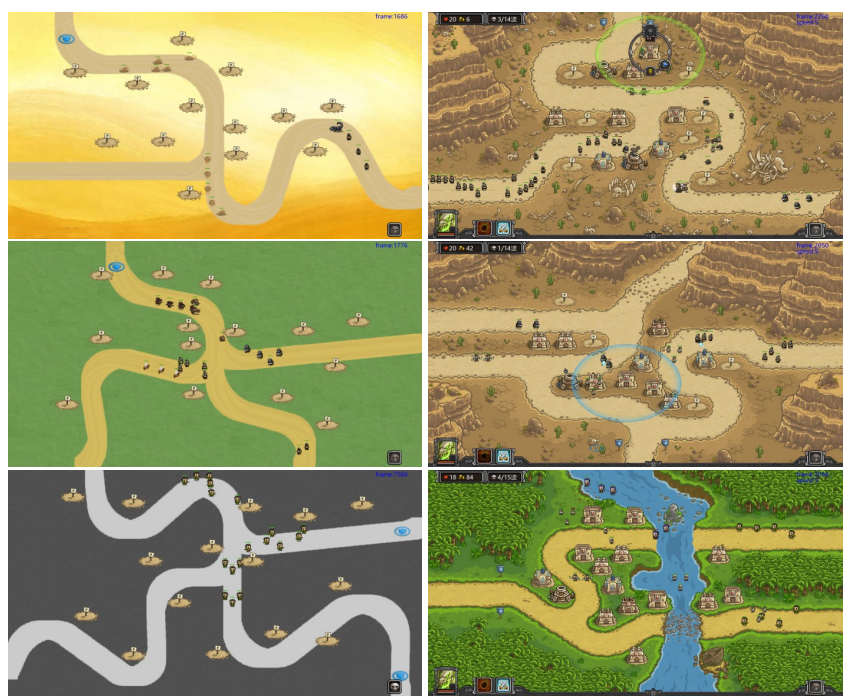

Figure 7: PCG levels 4, 6 and 8 (left) and corresponding levels from the original game (right). We preserve the number of roads and initial starting gold from the original reference level, but the road map, tower locations and monster sequences are new.

\section{RESULTS}

We present three complete levels created by PCG (see Figure 7). Videos of our automated agent playing these levels and outputting a difficulty curve over time can be viewed online. ${ }^{1}$ Each PCG level is modeled after a particular level from the original game (levels 4 , 6 and 8 respectively). We keep the initial starting gold and number of roads in the original level, but generate a new map and monster sequences. Alongside the outputs of each algorithm, we also report on the magnitude of computation required. Where exact times are given, tests were run on a single 6th-gen i7 processor. However, for the time-consuming monster wave generation and reinforcement learning-based playtesting, we can achieve an almost linear speedup by distributing work across multiple CPU cores (e.g. by using 10 cores we can produce a result ten times faster).

Over 10 runs of our PCG algorithm, we take on average $70 \mathrm{sec}-$ onds to generate a single map, including roads and tower point locations. Generating monster sequences is slower, taking on average 76 minutes using a single core.

\subsection{Road Generation}

The results of the road generation algorithm are shown in Figure 8 (left hand side). We create roads of a uniform width that monsters follow, and which constrain the placement of tower point locations.

\subsection{Tower Point Generation}

Tower points for three PCG levels are shown in Figure 8 (right hand side). We use the generated road network from the previous step as a starting point for placing tower point locations.

\footnotetext{
${ }^{1}$ https://youtu.be/qRcRM--8qa0
} 

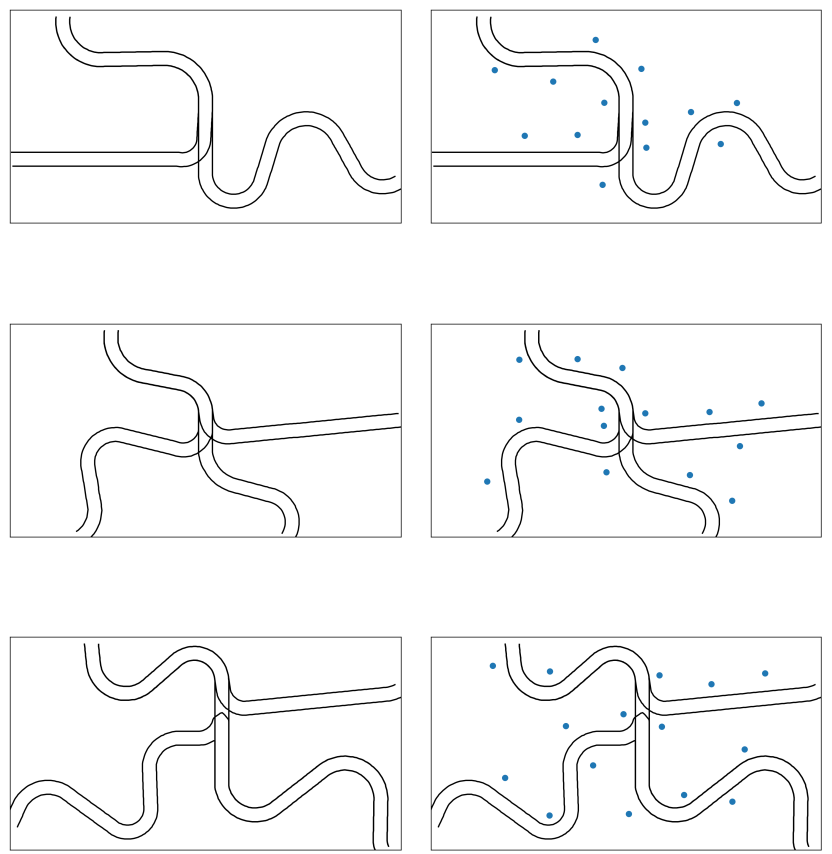

Figure 8: Generated road maps and tower points used in our three PCG levels.

\subsection{Monster Sequences}

An example monster sequence is shown in Figure 3. Highlighted are separate blocks of monsters that appear together in groups. In order to reduce the amount of searching required to find a reasonable solution, we use these blocks rather than individual monsters in our genetic algorithm to represent genes.

To evaluate how convincing the generated sequences are, we performed a simple Turing test with 13 staff at a game development company. Participants included a mixture of technical and non-technical staff, and most had not previously played the game KRF. We first showed each participant video examples of a humandesigned monster sequence and a PCG-designed sequence. Both sequences were displayed atop a human-designed background map, since we are currently unable to procedurally generate artwork of the same quality as that used in the human-designed levels. Each participant was then invited to watch six videos of monster sequences and rate each sequence on a scale from 1 to 5 , where the ratings indicate:

(1) Certainly PCG

(2) Probably PCG

(3) Unsure

(4) Probably human-designed

(5) Certainly human-designed

Based on the results, we gave each participant a total score:

$$
\text { Total Score }=\sum_{i=1}^{6} S_{i}, \text { where } S_{i}= \begin{cases}6-\text { rating }_{i} & \text { if PCG } \\ \operatorname{rating}_{i} & \text { otherwise }\end{cases}
$$

Three of the videos were human-designed and three were PCG, although this information was not divulged in advance to either the test subjects or the experimenter, making the test a double-blind trial.

\begin{tabular}{cccccccc}
\hline Participant & $S_{1}$ & $S_{2}$ & $S_{3}$ & $S_{4}$ & $S_{5}$ & $S_{6}$ & Total Score \\
\hline 1 & 1 & 5 & 5 & 1 & 1 & 1 & 14 \\
2 & 5 & 5 & 1 & 5 & 3 & 1 & 20 \\
3 & 1 & 5 & 1 & 5 & 5 & 1 & 18 \\
4 & 1 & 1 & 5 & 5 & 1 & 1 & 14 \\
5 & 5 & 5 & 1 & 1 & 5 & 5 & 22 \\
6 & 5 & 4 & 5 & 5 & 5 & 5 & 29 \\
7 & 1 & 1 & 5 & 5 & 5 & 5 & 22 \\
8 & 1 & 2 & 2 & 2 & 2 & 3 & 12 \\
9 & 1 & 1 & 2 & 4 & 2 & 4 & 14 \\
10 & 1 & 1 & 4 & 4 & 1 & 1 & 12 \\
11 & 1 & 3 & 1 & 2 & 3 & 2 & 12 \\
12 & 5 & 4 & 4 & 5 & 4 & 5 & 27 \\
13 & 2 & 5 & 5 & 1 & 1 & 3 & 17
\end{tabular}

Table 1: Scores from Turing test for human-designed and PCG Monster sequences

The mean score per participant is 17.9 (see Table 1 ), which is very close to the expected score of 18 that would be achieved by random guessing. This indicates that, as a group, our human test subjects were unable to distinguish reliably between human-designed and PCG sequences. However, participants 6 and 12, both of whom have prior experience with the game and achieved very high scores, described some slight flaws in the PCG sequences, notably that the timing between waves was noticeably longer than in the original game, and that the "rhythm" with which monsters were released was somewhat more predictable. We aim to fix these problems in the next version of our algorithm.

\subsection{Automated Playtesting}

We use reinforcement learning to playtest each PCG level. The RL algorithm has two purposes: first, it must find a winning strategy, which demonstrates that the level is passable. We define a winning strategy as one that does not allow any monsters to escape from the map. Second, we can use data from the RL agent to calculate a difficulty score for the entire level, which we can compare to that of the original levels (see Figure 9). This analysis could potentially be used by a human game designer or PCG algorithm as feedback on how to adjust the level. The game was played on the highest difficulty mode, which gives enemies extra hit points.

It is important to note that the reinforcement learning agent's difficulty assessment is inherently subjective, and is dependent on the strategy it uses to play the game. For example, we do not allow the agent to sell towers (in order to reduce the search space) and we do not reward the agent for saving money to buy more expensive towers; we also prioritize doing damage to enemy monsters as quickly as possible. A corollary of this action selection strategy is that the agent favors Archer Towers over other types (Figure 10), as they are the cheapest to build and generally the most effective against weaker monsters in the early stages of a level, 


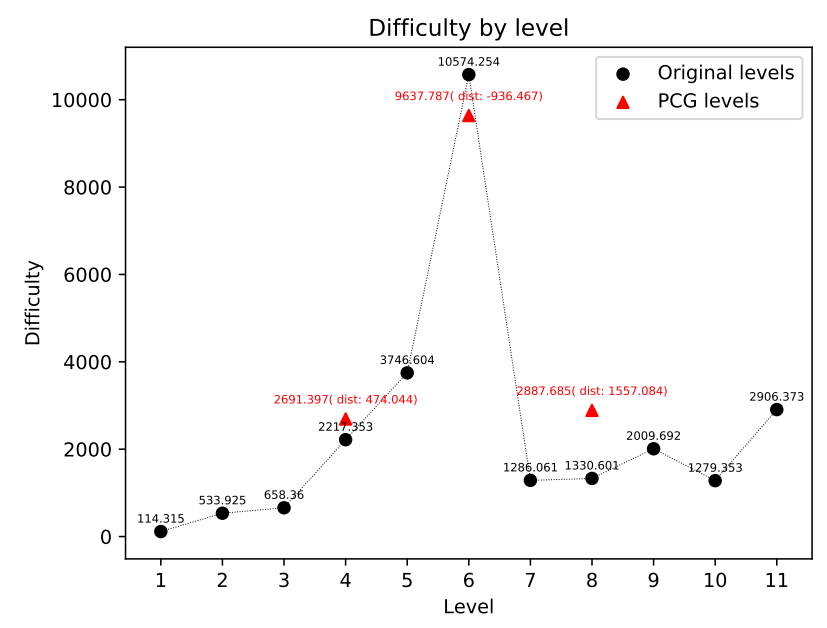

Figure 9: Total difficulty score for original 11 levels and PCG levels 4,6 and 8.

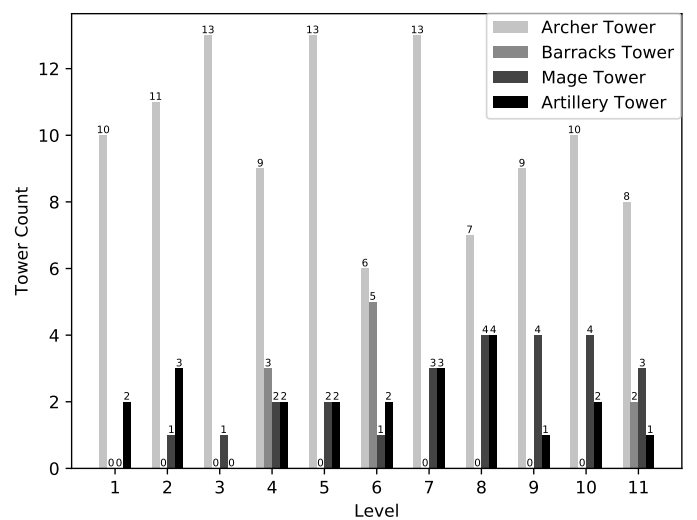

Figure 10: Distribution of tower types used by the flat Monte Carlo agent when playing the original 11 levels. The agent strongly favors the cheap Archer Towers over other types, although it diversifies its strategy somewhat in later levels when it finds the other towers are more effective.

and once built cannot be sold and exchanged for a different tower type. Simplistically speaking, this means levels easily won using a large proportion of Archer Towers are considered easy, while levels requiring more sophisticated tactics (such as using Barracks to obstruct monsters at vital choke points) are deemed hard. In practice, this may not correspond to a human player's perception of the game. In future research, the algorithm would need to be adjusted to mimic different human play styles.

\section{CONCLUSIONS}

In this paper, we have demonstrated a completely automated framework for procedurally generating Tower Defense game levels in the style of existing content, and using reinforcement learning to calculate a winning strategy and numerical difficulty assessment for each new level. We do this by dissecting tower defense levels into four fundamental building blocks (roads, tower points, monster sequences, and automated playtesting) and piecing together new levels in the manner of an assembly line. Our generated content is of high quality and is directly playable by humans. Our framework illustrates how modern AI techniques can successfully generate novel game content and assess its suitability for human play automatically, which has obvious applications in the video game industry. Future research could cover: investigating how to make the reinforcement learning agent more representative of human play styles, understanding how the AI's difficulty score is related to human perception, and how to create new level content de novo, without any human-designed levels as a reference.

\section{ACKNOWLEDGMENT}

The authors would like to thank Ironhide Studios, creators of the Kingdom Rush series, for many hours of fun playing and researching their tower defense games. We are also grateful to the anonymous reviewers, who provided many useful criticisms and suggestions.

\section{REFERENCES}

[1] R. Ariyadi, M. H. Purnomo, and S. M. Nugroho. 2014. Creep Offenses Evolution in Tower Defense Games using NSGA-II. In Proceedings of 8th International Conference on Information \& Communication Technology and Systems (ICTS), September 24, 2014, Surabaya, Indonesia. 153-157.

[2] P. Avery, J. Togelius, E. Alistar, and R. P. van Leeuwen. 2011. Computational Intelligence and Tower Defense Games. In Proceedings of the IEEE Congress on Evolutionary Computation (CEC 2011). New Orleans, USA.

[3] C. B. Browne, E. Powley, D. Whitehouse, S. Lucas, P. I. Cowling, P. Rohlfshagen, S. Tavener, D. Perez, S. Samothrakis, and S. Colton. 2012. A Survey of Monte Carlo Tree Search Methods. IEEE Transactions on Computational Intelligence and AI in Games 4, 1 (March 2012), 1-43.

[4] L. Cardamone, P. L. Lanzi, and D. Loiacono. 2015. TrackGen: An interactive track generator for TORCS and Speed-Dreams. Applied Soft Computing 28 (March 2015), 550-558.

[5] Candy Crush Saga Wiki contributors. 2019. Level. Retrieved March 26, 2019 from https://candycrush.fandom.com/wiki/Level?oldid=1355295

[6] F. de M. Silva, I. Borovikov, J. Kolen, N. Aghdaie, and K. Zaman. 2018. Exploring Gameplay With AI Agents. arXiv 1811.06962v1 (November 2018).

[7] F. de M. Silva, S. Lee, J. Togelius, and A. Nealen. 2018. Evolving Maps and Decks for Ticket to Ride. In Proceedings of Foundations of Digital Games conference (FDG'18), Malmö, Sweden, August 7-10, 2018.

[8] T. Duringer. 2017. Race for the Galaxy AI. Retrieved March 26, 2019 from http://www.templegatesgames.com/race-for-the-galaxy-ai/

[9] M. Epstein. 2016. How 'No Man's Sky' composes completely original music for every player. Retrieved March 26, 2019 from https://www.digitaltrends.com/ gaming/no-mans-sky-music/

[10] Gazza_N. 2019. gGuardian game for Windows. Retrieved March 26, 2019 from https://gazza-n.itch.io/gguardian

[11] T. T. Guan, Y. Y. Nan, C. K. On, J. Teo, and R. Alfred. 2013. Automated Evaluation for AI Controllers in Tower Defense Game Using Genetic Algorithm. In Communications in Computer and Information Science. Springer, 135-146.

[12] S. F. Gudmundsson, P. Eisen, E. Poromaa, A. Nodet, S. Purmonen, B. Kozakowski, R. Meurling, and L. Cao. 2018. Human-Like Playtesting with Deep Learning. In IEEE Conference on Computational Intelligence and Games (CIG'18), August 14-17, Maastricht, The Netherlands.

[13] M. Guzdial, J. Reno, J. Chen, G. Smith, and M. Riedl. 2018. Explainable PCGML via Game Design Patterns. arXiv 1809.09419v1 (September 2018).

[14] SHOKIZM INTERACTIVE. 2019. Tronix Defender game for Windows. Retrieved March 26, 2019 from https://shokizm.itch.io/tronix-defender

[15] I. Karth and A. M. Smith. 2018. Addressing the Fundamental Tension of PCGML with Discriminative Learning. arXiv 1809.04432v1 (September 2018).

[16] A. Liapis, G. N. Yannakakis, and J. Togelius. 2013. Sentient sketchbook: Computeraided game level authoring. In Proceedings of ACM Conference on Foundations of Digital Games, 2013. 
[17] M. Shaker, N. Shaker, and J. Togelius. 2013. Evolving Playable Content for Cut the Rope through a Simulation-based Approach. In Proceedings of the ninth AAAI Conference on Artificial Intelligence and Interactive Digital Entertainment, October 14-18, 2013, Boston, MA, USA. 72-78.

[18] N. Shaker, J. Togelius, and M. J. Nelson. 2016. Procedural Content Generation in Games: A Textbook and an Overview of Current Research. Springer.

[19] G. Smith, J. Whitehead, and M. Mateas. 2011. Tanagra: Reactive Planning and Constraint Solving for Mixed-Initiative Level Design. IEEE Transactions on Computational Intelligence and AI in Games 3, 3 (September 2011), 201-215.

[20] ShotX Studio. 2019. Dead Ground game for Windows and Linux. Retrieved March 26, 2019 from https://shotx.itch.io/deadground

[21] A. Summerville, S. Snodgrass, M. Guzdial, C. Holmgard, A. K. Hoover, A. Isaksen, A. Nealen, and J. Togelius. 2017. Procedural Content Generation via Machine Learning (PCGML). arXiv 1702.00539v1 (February 2017).

[22] R. Sutoyo, D. Winata, K. Oliviani, and D. M. Supriyadi. 2015. Dynamic Difficulty Adjustment in Tower Defence. In Proceedings of International Conference on Computer Science and Computational Intelligence (ICCSCI 2015), August 24-26, 2015, fakarta, Indonesia. 435-444.

[23] R. S. Sutton and A. G. Barto. 2018. Reinforcement Learning: An Introduction (2nd ed.). MIT Press, Cambridge, MA.

[24] V. Volz, J. Schrum, J. Liu, S. M. Lucas, A. Smith, and S. Risi. 2018. Evolving Mario Levels in the Latent Space of a Deep Convolutional Generative Adversarial Network. In Proceedings of the Genetic and Evolutionary Computation Conference (GECCO 2018). Kyoto, Japan.

[25] G. N. Yannakakis and J. Togelius. 2018. Artificial Intelligence and Games. Springer, New York. 